Classification

Physics Abstracts

$73.30-73.60-79.20 \mathrm{~F}-79.60$

\title{
Stabilité de l'émission photoélectrique de couches minces d'argent recouvertes de césium oxydé
}

\author{
L. Gautreau et A. Septier
}

Laboratoire de Physique du Vide, ERA 660, C.N.A.M., 292, rue Saint-Martin, 75141 Paris Cedex 03, France

(Reçu le 28 uvril 1983, révisé le 12 janvier 1984, accepté le 13 février 1984)

\begin{abstract}
Résumé. - Par une série de cycles alternés d'évaporation de Cs suivie d'une introduction contrôlée d'oxygène dans l'enceinte ultravide, on forme une mince couche $\mathrm{Cs}-\mathrm{O}$ qui abaisse le travail de sortie de Ag polycristallin aux environs de $1 \mathrm{eV}$. Mais le seuil photoélectrique $\lambda_{\mathrm{s}} \approx 1,2 \mu \mathrm{m}$ ainsi obtenu n'est pas stable, et se déplace lentement vers les plus courtes longueurs d'onde. Toutefois, les valeurs du seuil initial et de la sensibilité spectrale $S(\lambda)$ de la photocathode peuvent être réobtenues aisément par introduction dans l'enceinte à ultravide d'une très faible quantité d'oxygène. Des analyses Auger de la couche superficielle Cs-O ont permis de montrer que cette couche est formée d'atomes adsorbés et non d'oxyde de Cs, que son épaisseur est de l'ordre de la monocouche, et que la variation des pics relatifs à $\mathrm{Cs}$ et $\mathrm{O}$ pendant le vieillissement, correspond à un appauvrissement en oxygène qui peut être compensé par régénération.
\end{abstract}

\begin{abstract}
Controlled oxidation of successive Cs layers evaporated in sequence onto a polycristallin Ag surface decreases the work function down to $1 \mathrm{eV}$. Unfortunately, the corresponding wavelength threshold $\lambda_{\mathrm{s}} \approx 1.2 \mu \mathrm{m}$ is not stable and moves slowly towards lower values. However initial threshold and spectral sensitivity $S(\lambda)$ values can be fully recovered by injecting a tiny amount of oxygen in the UHV chamber. AES studies, performed with new, aged and regenerated Cs-oxide layers respectively, allowed to detect very small variations in the oxygen concentration and to show that the Cs-O layer is not made of Cs-oxide but of Cs and $\mathrm{O}$ adsorbed atoms, with a thickness corresponding to about one monolayer.
\end{abstract}

\section{Introduction.}

L'émission photoélectrique de couches polycristallines d'argent ou d'aluminium recouvertes d'un film très mince de césium est parfaitement stable, lorsque les expériences sont effectuées dans un ultravide très propre, sous une pression résiduelle inférieure à $10^{-10}$ torr, $[1,2]$, avec un seuil photoélectrique situé au voisinage de $\lambda_{\mathrm{s}} \approx 0,8 \mu \mathrm{m}$. Nous avons réalisé des photocathodes très sensibles dans l'infrarouge proche [2] en recouvrant l'argent d'un film très mince obtenu à température ambiante par des cycles alternés de dépôt puis d'oxydation de césium, répétés jusqu'à obtention d'une sensibilité maximum dans l'infrarouge et d'un travail de sortie voisin de $1 \mathrm{eV}$. Mais l'expérience montre que les propriétés émissives des surfaces d'argent ainsi activées ne sont pas stables dans le temps, et on assiste à une diminution lente de la longueur d'onde seuil $\lambda_{\mathrm{s}}$ de $1,20 \mu \mathrm{m}$ à $1 \mu \mathrm{m}$ en quelques jours.

Nous avons cherché [3] à reproduire ces expériences dans un bâti à ultravide équipé d'un analyseur Auger, pour étudier le phénomène de vieillissement des photocathodes, puis tenté de les régénérer, et enfin de suivre l'évolution de la composition chimique de la couche d'oxyde de césium, responsable de la dégradation des propriétés photoémissives. On ignore la composition exacte de la couche d'oxyde de césium, ce métal étant capable de former plusieurs types d'oxydes. Il semble $[4,5]$ que l'oxyde dominant soit $\mathrm{Cs}_{2} \mathrm{O}$, mais la présence d'inclusions ou d'îlots de $\mathrm{Cs}_{11} \mathrm{O}_{3}$ semble vraisemblable.

L'analyse Auger ne nous permettra d'atteindre que les taux moyens respectifs de $C s$ et $O$ dans la couche, et non la composition vraie, mais l'évolution respective des hauteurs de pics caractéristiques de ces deux éléments au cours du temps devrait permettre d'élucider en partie le processus de dégradation.

Une meilleure compréhension du phénomène de vieillissement des photocathodes recouvertes d'une couche d'oxyde de césium - et en particulier les photocathodes à affinité négative [6] faites à partir 
d'un monocristal semiconducteur ( $\mathrm{Si}$ ou $\mathrm{AsGa}$ ) pour lesquelles des variations importantes de sensibilité ont été également signalées $[7,8]$ devraient permettre d'en améliorer les techniques de fabrication.

En outre, l'analyse Auger devrait nous renseigner sur l'épaisseur et la nature de la couche de césiumoxygène formée à la surface de l'argent.

\section{Expériences.}

2.1 Dispositif eXPÉRIMENTAL. - Le dispositif expérimental est représenté schématiquement sur la figure 1. L'enceinte à ultravide, de $30 \mathrm{~cm}$ de diamètre et $50 \mathrm{~cm}$ de hauteur, comporte outre le système de pompage (pompe ionique et sublimateur de titane) :

- un spectromètre Auger constitué par un analyseur électrostatique de type CMA et un canon axial,

- un porte-échantillon tournant avec déplacements possibles en $x, y$ et $z$,

- deux évaporateurs chauffés par effet Joule pour l'obtention des couches d'argent et des dépôts de césium,

- une balance à quartz pour la mesure d'épaisseur des dépôts métalliques,

- un système d'introduction d'oxygène,

- divers hublots en silice fondue,

- une électrode en forme d'anneau destinée à capter le courant électronique émis par les photocathodes.

Un prisme en silice fondue de $20 \times 20 \times 20 \mathrm{~mm}^{3}$ est fixé sur le porte-échantillon, l'axe vertical de rotation de ce dernier étant tangent à l'une des faces carrées du dièdre droit, sur laquelle sera déposée la couche d'argent mince.

A l'extérieur de l'enceinte un ensemble optique, formé d'une source à ruban de tungstène, un monochromateur à réseau, et un polariseur permet d'éclai- rer la photocathode avec un faisceau parallèle quasi monochromatique $(\Delta \lambda \simeq 8 \mathrm{~nm})$. Une photodiode au silicium, préalablement étalonnée à l'Institut National de Métrologie du CNAM par comparaison avec une thermopile de caractéristiques connues, peut être introduite sur le trajet du faisceau, pour déterminer la puissance $W_{\lambda}$ transportée par le faisceau ce qui, après mesure du courant émis $i_{\mathrm{E}}$, permet de déterminer la sensibilité $S_{\lambda}$ de la photocathode :

$$
S_{\lambda}=\frac{i_{\mathrm{E}}}{W_{\lambda}}\left(\mathrm{A} \mathrm{W}^{-1}\right) \text {. }
$$

Le support des couches, constitué par le prisme, rend possible l'excitation dans le film d'argent d'ondes de plasma de surface par la méthode ATR [9], en vue d'accroître la sensibilité $S_{\lambda}$. La face supérieure du prisme, et l'autre face du dièdre droit sont recouvertes d'une couche épaisse d'argent, en contact électrique avec la base du porte-échantillon, ellemême isolée du manipulateur, ce qui permet la mesure du courant photoélectrique issu de la cathode isolée de l'enceinte.

La pression limite dans l'enceinte à vide a pu être abaissée à $5 \times 10^{-11}$ torr grâce à :

- des cycles de dégazages des parois et de la pompe ionique à $T=250^{\circ} \mathrm{C}$ pendant plusieurs semaines,

- un dégazage des électrodes et des parois internes de l'analyseur Auger par bombardement électronique effectué à l'aide du canon défocalisé au maximum et émettant un courant aussi élevé que possible (plusieurs $\mathrm{mA}$ ) sous une tension de $3 \mathrm{kV}$,

- un dégazage soigné des évaporateurs, portés à des températures légèrement inférieures aux points de fusion respectifs de $\mathrm{Ag}$ et $\mathrm{Cs}$.

2.2 FABRICATION DES COUCHES ACTIVÉES AU CÉSIUM. On évapore sur le prisme une couche mince d'argent d'épaisseur $50 \mathrm{~nm}$ en $2 \mathrm{~min}$. L'analyse Auger effectuée

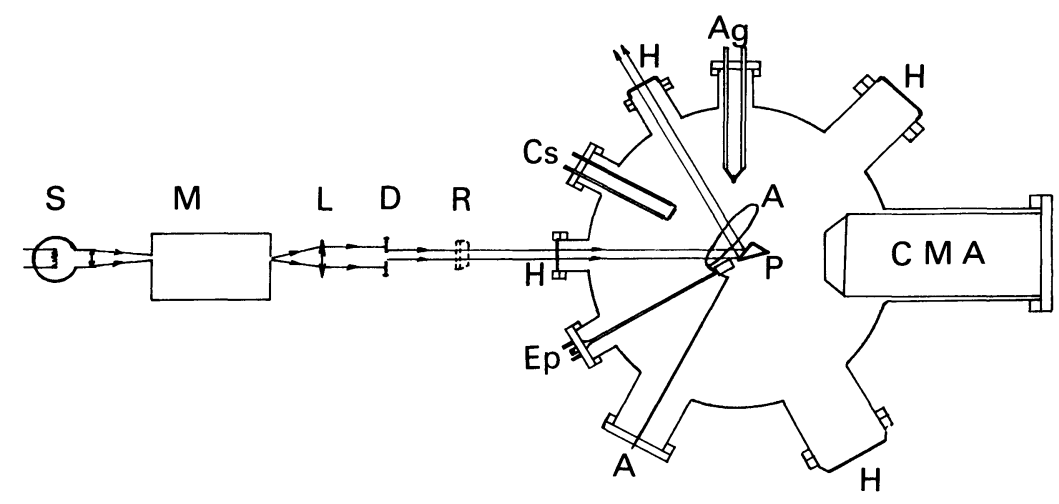

Fig. 1. - Dispositif expérimental. CMA : analyseur Auger - Ag : évaporation d'argent - Cs : évaporateur de césium - $E_{p}$ : balance à quartz $-\mathrm{S}:$ source lumineuse $-\mathrm{A}:$ monochromateur $-\mathrm{L}:$ lentille $-\mathrm{D}:$ diaphragme $-\mathrm{R}:$ photodiode escamotable $-\mathbf{H}$ : hublots en silice fondue $-\mathbf{P}$ : prisme en silice $-\mathrm{A}$ : anode.

[Experimental set-up. CMA : Auger analyser - Ag: Ag evaporator - Cs : Cs evaporator $-\mathrm{E}_{\mathrm{p}}:$ quartz thickness monitor $-\mathbf{S}:$ light source $-\mathbf{M}:$ monochromator $-\mathrm{L}:$ Lens $-\mathrm{D}:$ diaphragm $-\mathbf{R}:$ photodiode $-\mathrm{H}:$ fused silica windows $\mathrm{P}$ : silica prism - A : collecting anode.] 
sur la surface de l'argent montre une absence totale de pics parasites : aucune impureté n'est décelable, dans les limites de sensibilités de l'analyseur, cependant capable de mettre en évidence une très faible fraction de monocouche.

Avant évaporation du césium, la source (SAES Getter, Milano) est de nouveau dégazée, un cache mobile étant interposé entre l'évaporateur et la cible d'argent. Le film d'argent est alors éclairé par un faisceau de longueur d'onde $\lambda_{i}=0,5 \mu \mathrm{m}$ puis on procède à une évaporation très lente du $\mathrm{Cs}$ - et on mesure l'émission photoélectrique. On accroît ensuite progressivement $\lambda_{\mathrm{i}}$, à mesure que le seuil $\lambda_{\mathrm{s}}$ se déplace vers le rouge. L'évaporation est arrêtée lorsque l'émission électronique atteint son maximum. Toutefois, la quantité de Cs évaporée n'est pas critique, car lorsque le maximum d'émission est dépassé - avant l'arrêt de l'évaporation - l'émission photoélectrique croît de nouveau et revient d'elle-même au maximum. Ce phénomène s'explique aisément, si on admet que l'émission maximum est obtenue lorsque la surface a un travail de sortie minimum, correspondant à une fraction de monocouche de Cs adsorbée sous forme ionique [10], les ions Cs étant fortement liés au réseau d'Ag, alors que les atomes en surplus s'évaporent rapidement. (A $T=300 \mathrm{~K}$ la pression de vapeur saturante de Cs est d'environ $10^{-6}$ torr.)

Dans nos expériences, le seuil photoélectrique se situe à $\lambda_{\mathrm{s}} \simeq 0,75 \mu \mathrm{m}$, correspondant à un travail de sortie de $1,7 \mathrm{eV}$, légèrement inférieur à celui du $\mathrm{Cs}$ massif.

Après cette évaporation, la sensibilité photoélectrique $S(\lambda)$ n'évolue pratiquement pas au cours du temps. On note toutefois après 10 jours une légère augmentation de $\lambda_{s}$, certainement due à un début d'oxydation du Cs par l'oxygène de l'atmosphère résiduelle.

Cette stabilité confirme l'excellente qualité du vide obtenu dans l'enceinte. En effet, elle n'est atteinte que pour des pressions limites très inférieures à $10^{-10}$ torr, et si on évite d'utiliser l'analyseur Auger avant - ou après - l'évaporation du Cs. Ce dernier point montre que l'analyseur en fonctionnement émet un flux de gaz désorbés suffisant pour polluer la surface et en accroître le travail de sortie, bien que l'analyse Auger soit incapable de déceler la moindre impureté à la surface de l'argent.

L'analyse Auger de la surface d'argent césié permet de voir avec une très bonne sensibilité les pics caractéristiques du césium et de l'argent, sans aucun pic parasite. Toutefois, l'observation n'est possible qu'en réduisant le courant électronique $(I<0,1 \mu \mathrm{A})$ afin de ne pas évaporer la couche de Cs par un échauffement local trop important, le support de la couche d'argent étant très mauvais conducteur thermique.

2. 3 OBTENTION D'UNE COUCHE DE CÉSIUM OXYDÉ. Par suite de l'effet nocif de l'analyse Auger sur l'émission photoélectrique, la fabrication des couches acti- vées à l'oxyde de Cs s'effectue sans analyse. Après obtention de la couche de Cs stable, on introduit de l'oxygène très pur jusqu'à obtention d'une pression de $2 \times 10^{-10}$ torr (la pression initiale étant de $5 \times 10^{-11}$ torr). La jauge à ionisation est éteinte, pour éviter toute lumière parasite, et on éclaire la couche avec un rayonnement de longueur d'onde $\lambda_{\mathrm{i}}=0,65 \mu \mathrm{m}$. L'émission photoélectrique croît lentement. Au bout de $20 \mathrm{~min}$, elle passe par un maximum, correspondant à une exposition à l'oxygène de 0,24 Langmuir. On arrête le flux d'oxygène lorsque ce maximum est atteint. Une telle exposition à l'oxygène accroît la longueur d'onde seuil $\lambda_{\mathrm{s}}$ et la sensibilité $S_{\lambda}$ pour toutes les longueurs d'ondes. Par contre, l'action d'une nouvelle évaporation de césium est plus complexe : $S_{\lambda}$ augmente autour de $\lambda=0,65 \mu \mathrm{m}$, mais le seuil recule légèrement (Fig. 2). Par suite, pour optimiser une photocathode, on utilise $\lambda \approx \lambda_{\mathrm{s}}$ en présence d'oxygène et on arrête l'introduction de gaz lorsqu'on atteint le maximum d'émission électronique à cette longueur d'onde. Puis on revient à $\lambda=0,65 \mu \mathrm{m}$ pour contrôler le dépôt de césium, en recherchant de nouveau le maximum de courant émis. On procède ainsi à des cycles alternés évaporation-oxydation de Cs. Dans tous les cas, 9 à 10 cycles sont suffisants pour que $\lambda_{\mathrm{s}}$ atteigne sa valeur maximum. Si l'on continuait le traitement, on accroîtrait l'épaisseur de la couche sans modifier pratiquement $\lambda_{\text {s. }}$.

Dans tout ce qui suit, nous étudierons les couches d'épaisseur minimum pour lesquelles $\lambda_{\mathrm{s}} \approx 1,2 \mu \mathrm{m}$.

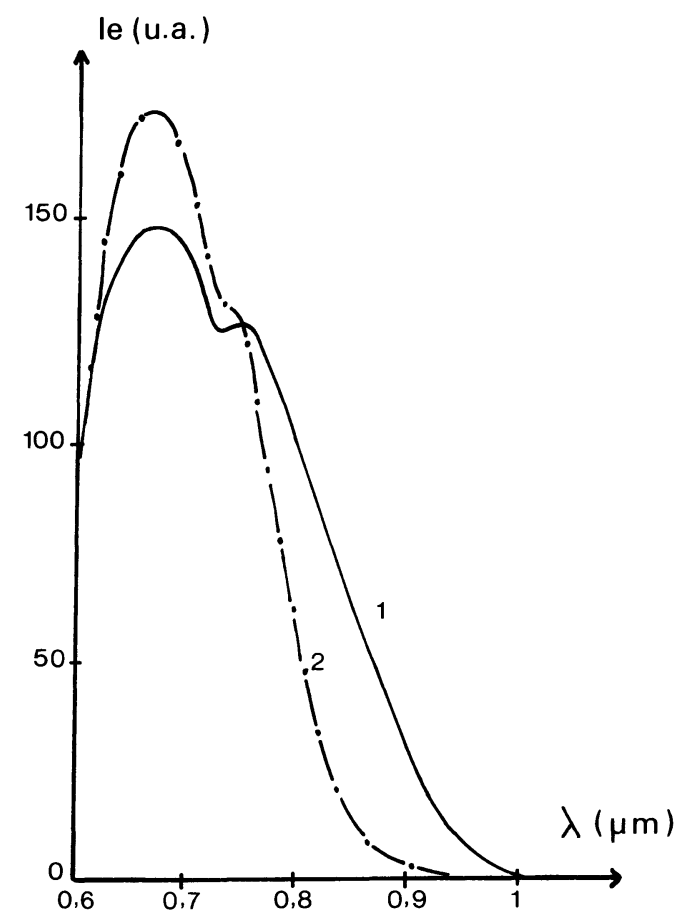

Fig. 2. - Emission photoélectrique de la photocathode (unités arbitraires) (1) après la fin du $3^{\mathrm{e}}$ cycle Cs-O - (2) après la $4 \mathrm{e}$ évaporation de Cs (début du $4^{\mathrm{e}}$ cycle).

[Photoelectric emission (arbitrary units) of the Ag photocathode (1) after the 3rd adsorption of oxygen - (2) after the 4 th evaporation of Cs.] 
2.4 RÉSULTATS : SENSIBILITÉ SPECTRALE DES COUCHES ACTIVÉES. - On obtient finalement des photocathodes sensibles dans l'IR jusqu'à $\lambda_{\mathrm{s}}=1,2 \mu \mathrm{m}$, dont la sensibilité spectrale présente un palier entre 0,7 et $0,9 \mu \mathrm{m}$. La figure 3 représente l'évolution de cette sensibilité $S_{\lambda}$ en cours de fabrication : après la première évaporation de césium, puis après les $2 \mathrm{e}, 4 \mathrm{e}$, $6^{\mathrm{e}}$ et $9^{\mathrm{e}}$ cycles d'oxydation.

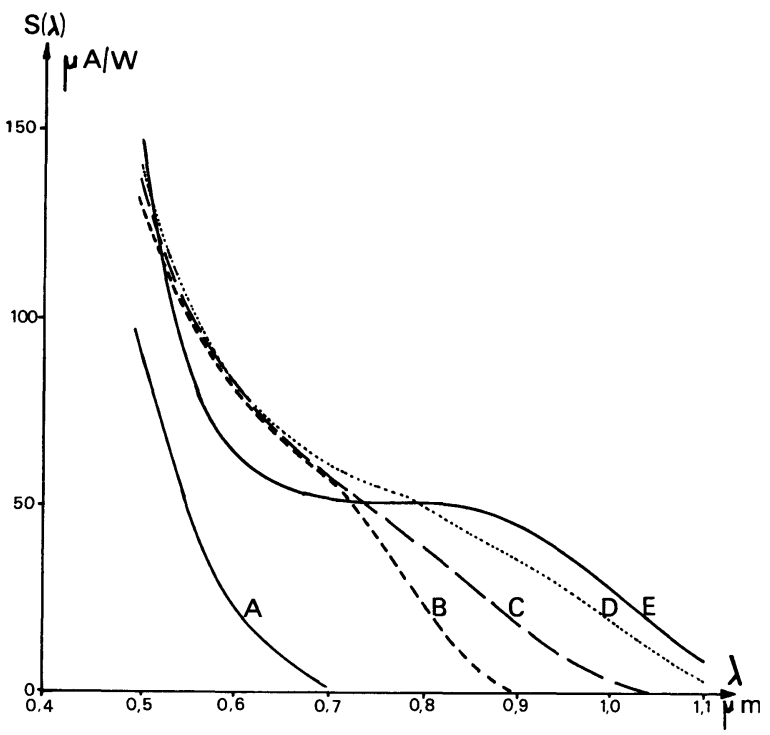

Fig. 3. - Evolution de la sensibilité spectrale des photocathodes pendant la réalisation. $\mathrm{A}: \mathrm{Ag}-\mathrm{Cs}-\mathrm{B}$ : après $2^{e}$ traitement $\mathrm{Cs}+\mathrm{O}_{2}-\mathrm{C}$ : après $4^{\mathrm{e}}$ traitement $-\mathrm{D}$ : après $6^{\mathrm{e}}$ traitement $-\mathrm{E}$ : après $9^{\mathrm{e}}$ et dernier traitement.

[Evolution of the spectral sensitivity $S_{\lambda}$ during the photocathode activation - $\mathrm{A}: \mathrm{Ag}+\mathrm{Cs}-\mathrm{B}:$ at the end of the $2^{\text {nd }} \mathrm{Cs}+\mathrm{O}_{2}$ cycle $-\mathrm{C}:$ after the 4 th cycle $-\mathrm{D}:$ after the 6 th cycle $-\mathrm{E}:$ after the 9 th cycle.]

La figure 4 montre l'évolution du travail de sortie $W_{\mathrm{s}}$ des couches émissives en fonction de la durée cumulée des oxydations sous pression dynamique d'oxygène de $2 \times 10^{-10}$ torr. L'oxydation totale dure $70 \mathrm{~min}$. environ, ce qui correspond à une quantité d'oxygène inférieure à 1 Langmuir. Cette valeur est très inférieure à la fois à celle indiquée par Uebbing et James [11] et à celle observée dans notre laboratoire par G. Hincelin et A. Septier [2], toutes deux de l'ordre de 5 à 6 Langmuir. Il semble donc que la vitesse de formation de la couche optimisée d'oxydes de césium soit fonction du dispositif expérimental (volume de l'enceinte, présence ou non d'ions oxygène lorsque la surface sensible est - comme dans notre cas - située à proximité de la pompe ionique).

Malgré toutes les précautions prises pour le dégazage de l'analyseur Auger, la mise en fonction de cet analyseur pour obtenir des spectres à chacun des stades de fabrication de la surface sensible conduit à l'obtention de photocathodes ayant des sensibilités $S_{\lambda}$ nettement plus faibles dans l'IR - mais par contre plus élevées dans le visible - que celles des photo-

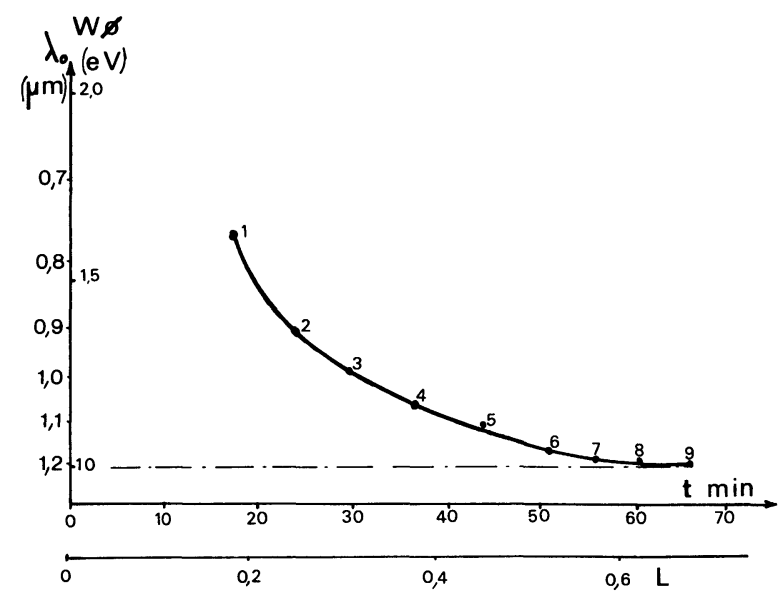

Fig. 4. - Evolution du seuil photoélectrique et du travail de sortie en fonction de la quantité d'oxygène (en Langmuirs, $1 \mathrm{~L}=10^{-6}$ torr.S). $1,2,3, \ldots, 9$ : fin de chacun des traitements $\mathrm{Cs}+\mathrm{O}_{2}$

[Evolution of the photoelectric threshold, and of the work function as a function of the total oxygen exposure (1 Langmuir $=10^{-6}$ torr.s). $1,2,3, \ldots, 9$ : end of each cycle Cs evaporation + controlled oxidation.]

cathodes réalisées sans analyse. Il faut 10 cycles d'évaporation-oxydation pour obtenir la valeur maximum de $\lambda_{\mathrm{s}}$, mais celle-ci ne peut dépasser $1,15 \mu \mathrm{m}$ et l'allure de la courbe finale donnant $S_{\lambda}$ est très différente de celle obtenue sans analyses Auger. On peut donc supposer que le chauffage du filament du canon et le bombardement des électrodes internes par une partie du faisceau électronique continuent de produire des atomes polluants en quantité non détectable sur les spectres, mais suffisante pour modifier profondément l'émission photoélectrique de la surface, en particulier par élévation du travail de sortie.

En outre, il faut noter que, même sans analyse Auger, les valeurs absolues de $S_{\lambda}$ obtenues dans notre appareillage n'ont jamais pu atteindre celles obtenues par G. Hincelin [2] dans un dispositif de plus petit volume et de conception différente travaillant à la même pression limite de $5 \times 10^{-11}$ torr. Ceci pourrait être interprété en supposant la présence dans l'atmosphère résiduelle de notre enceinte, de traces de gaz polluants, qui n'existeraient pas dans celle de G. Hincelin. Des analyses de gaz résiduels n'ont pas été envisagées, car à ces pressions très basses, l'analyseur de gaz lui-même est source de pollution et on ne peut savoir si les pics observés correspondent à des gaz présents dans l'enceinte en l'absence d'analyseur.

Il semble que $\mathrm{CO}, \mathrm{CO}_{2}$ ou tout autre gaz carboné présent dans l'atmosphère résiduelle ne s'adsorbe pas sur la surface car les spectres Auger ne montrent aucune trace de carbone.

Si un dégazage du canon produit de l'hydrogène, ce gaz ne peut être détecté. Mais, bien que l'adsorption d'atomes d'hydrogène sur une surface recouverte de césium soit très difficile [12] on peut.supposer que ce 
gaz puisse être responsable d'une faible remontée du travail de sortie - et donc d'une diminution de $\lambda_{\mathrm{s}}$.

\subsection{EMISSION RENFORCÉE PAR EXCITATION D'ONDES} DE PLASMA DE SURFACE. - La couche support d'argent étant évaporée sur un prisme, il est possible de l'éclairer à travers le prisme en lumière monochromatique non polarisée. Pour un certain angle d'incidence $\theta_{\mathrm{i}}$ il y a absorption importante de la lumière incidente par suite de l'excitation d'une onde de plasma de surface à l'interface argent-vide. L'angle $\theta_{\mathrm{i}}$ est fonction de $\lambda$. Par suite du renforcement considérable de l'énergie électromagnétique absorbée dans la zone voisine de la surface, l'émission photoélectrique est accrue par un facteur important. La figure 5 donne la sensibilité $S_{\lambda}$ de la photocathode correspondant au maximum d'excitation de l'onde de surface, et le facteur multiplicatif obtenu par comparaison à la sensibilité $S_{\lambda}$ de la figure 2. Ce facteur, surtout important dans l'IR, dépasse 60 vers $0,85 \mu \mathrm{m}$ et 50 à $1,1 \mu \mathrm{m}$.

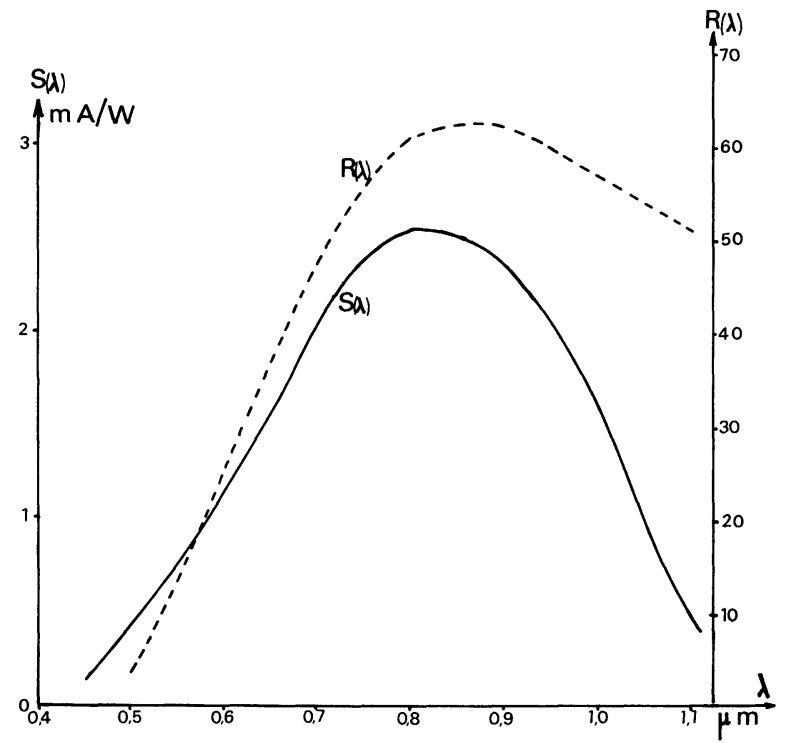

Fig. 5. - Sensibilité spectrale $S_{\lambda}$ d'une photocathode optimisée avec excitation de plasmons de surface - et coefficient de renforcement de l'émission $R_{\lambda}$.

[Photocathode spectral sensitivity $S_{\lambda}$ enhanced by surface plasma wave excitation and emission enhancement coefficient $R_{\lambda}$.]

\section{Vieillissement et régénération des photocathodes.}

3.1 EMISSION DES COUCHES. - Après fabrication d'une couche activée à l'oxyde de césium de sensibilité maximum - donc sans analyse Auger - on assiste à un recul progressif vers le visible du seuil $\lambda_{\mathrm{s}}$, qui passe de 1,2 à $1,1 \mu \mathrm{m}$ en $40 \mathrm{~h}$, recul accompagné d'un renforcement de $S_{\lambda}$ vers $\lambda=0,75 \mu \mathrm{m}$. La figure 6 représente cette évolution (courbes $\mathrm{E}$ et $\mathrm{F}$ ) qui ressemble à celle observée durant la fabrication de la surface sensible après chaque cycle évaporation-

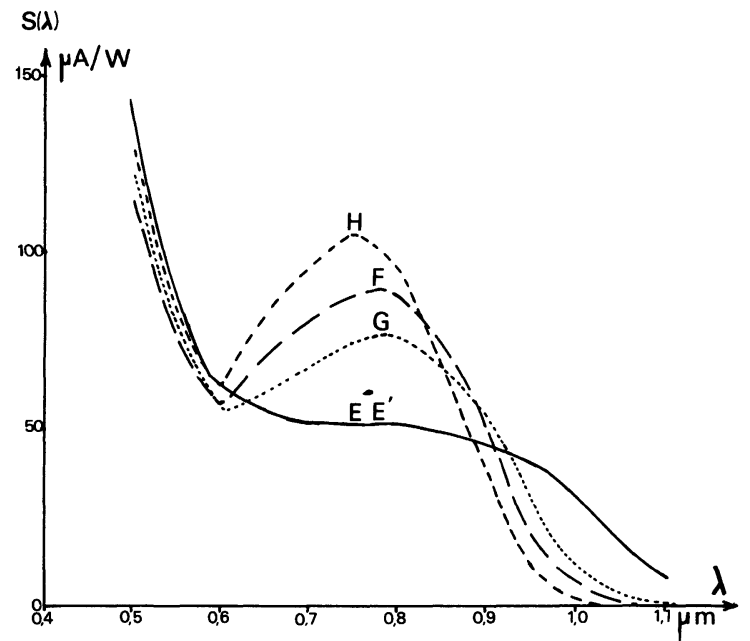

Fig. 6. - Vieillissement d'une photocathode : évolution de la sensibilité spectrale - E : $S_{\lambda}$ d'une cathode neuve (identique à la courbe $\mathrm{E}$ de la Fig. 2) - F : $20 \mathrm{~h}$ après $E-E^{\prime}$ : après régénération par l'oxygène $-G: 16 h$ après $E^{\prime}-H: 40 h$ après $E^{\prime}$.

[Photocathode aging : evolution of the spectral sensitivity $-\mathrm{E}$ : freshly activated $\mathrm{Ag}$ surface (identical to curve $\mathrm{E}$ of Fig. 2) $-F: 20 \mathrm{~h}$ later $-\mathrm{E}^{\prime}$ : after regeneration by a new $\mathrm{O}_{2}$ exposure $-\mathrm{G}: 16 \mathrm{~h}$ after $\mathrm{E}^{\prime}-\mathrm{H}: 40 \mathrm{~h}$ after $\mathrm{E}^{\prime}$.]

oxydation lorsqu'on évapore Cs sur la couche oxydée précédente.

On peut donc supposer que le vieillissement observé des couches d'oxyde de césium est dû à un enrichissement local en césium provoqué par le départ d'une certaine quantité d'oxygène.

Cet appauvrissement en oxygène est confirmé par l'expérience suivante : après $20 \mathrm{~h}$ de vieillissement dans le vide résiduel à $p \simeq 5 \times 10^{-11}$ torr, une introduction d'une très faible quantité d'oxygène dans l'enceinte $\left(2 \times 10^{-10}\right.$ torr pendant $2,5 \mathrm{~min}$. soit 0,03 Langmuir) permet de régénérer la photocathode et de réobtenir la sensibilité d'origine (courbe $\mathrm{E}^{\prime}$ de la Fig. 6). Mais, après régénération, l'évolution reprend : courbes $\mathbf{G}$ et $\mathbf{H}$ obtenues respectivement après 16 et $40 \mathrm{~h}$.

Les expériences de vieillissement n'ayant duré que $40 \mathrm{~h}$, nous ne pouvons affirmer que l'évolution existe encore après ce délai.

3.2 ANalyses Auger. - Un spectre de référence est obtenu avec la couche d'argent de $50 \mathrm{~nm}$ fraîchement évaporée, avant dépôt de $\mathrm{Cs}$, dans les conditions suivantes : $I_{\mathrm{p}}=10^{-7} \mathrm{~A}, V_{\mathrm{p}}=2000 \mathrm{~V}$. Un petit pic Cs est visible sur le spectre, à une énergie de $50 \mathrm{eV}$ : il est dû au dépôt sur l'argent d'atomes de Cs présents sur les parois et dans l'atmosphère de l'enceinte, à la suite des évaporations précédentes. Aucune trace d'impureté n'est visible. Un second spectre est tracé après la première évaporation de Cs et stabilisation de l'émission photoélectrique.

La présence du césium n'entraîne qu'une diminution d'environ $10 \%$ du pic $\mathrm{Ag}(358 \mathrm{eV})$, ce qui signifie 
que la couche de Cs est très mince. Malgré cela le pic Cs $(50 \mathrm{eV})$ est très visible sur le spectre (Fig. 7), mais avec une amplitude 10 fois plus faible que le pic correspondant au support d'argent $(\mathrm{Ag}, 352 \mathrm{eV})$. D'autres pics Cs existent à 562 et $574 \mathrm{eV}$; ils sont très faibles.

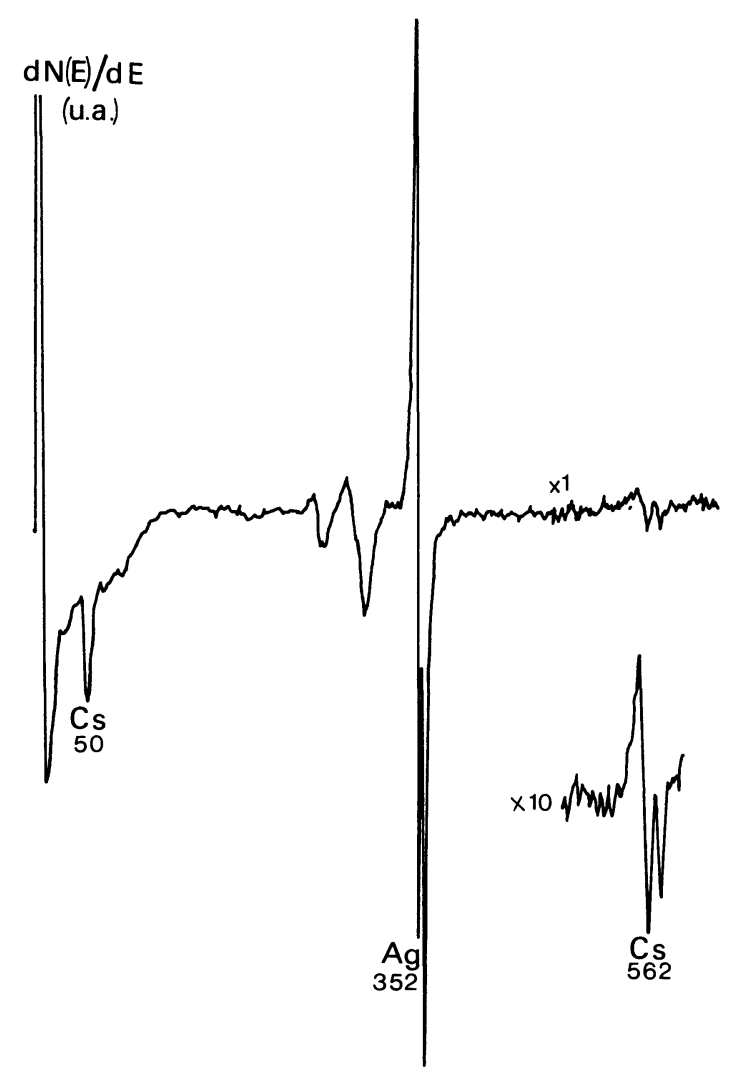

Fig. 7. - Spectre Auger de la couche d'argent césié à sensibilité $S_{\lambda}$ maximum. $(V=2000 \mathrm{~V}-I=0,1 \mu \mathrm{A}-$ $V$ modulation $=4 \mathrm{~V}$ - Faisceau défocalisé.)

[Auger spectrum of a Cs activated surface. (Operating conditions : $V=2000 \mathrm{~V}-I=0.1 \mu \mathrm{A}-V$ modulation $=4 \mathrm{~V}-$ defocused beam.) $]$

Après la première "oxydation " de la couche initiale de Cs, les pics Cs $(50 \mathrm{eV})$ et Cs $(562 \mathrm{eV})$ ne varient pas, et on voit apparaître un très faible signal caractéristique de l'oxygène à une énergie de $516 \mathrm{eV}$, avec un rapport signal/bruit de l'ordre de 2 à 3 seulement.

L'amplitude des différents pics Cs et $\mathrm{O}$ croît régulièrement après chaque cycle d'évaporation-oxydation. Après 9 cycles, soit $60 \mathrm{~min}$ d'oxydation à $p=2 \times 10^{-10}$ torr, le signal Cs $(50 \mathrm{eV})$ est multiplié par 4 environ, et le signal $\mathrm{O}(516 \mathrm{eV})$ par 8 (Fig. 8).

Par contre, le pic Ag (352 eV) dû aux électrons de la couche support ayant traversé la couche superficielle Cs-O varie peu au cours des traitements successifs. Entre l'évaporation de la première couche de Cs et la $9^{\mathrm{e}}$ oxydation, il décroît seulement de $10 \%$ environ. Si l'on admet pour l'oxyde de Cs, et des électrons de $352 \mathrm{eV}$ un libre parcours d'environ $1 \mathrm{~nm}$. Cette obser-

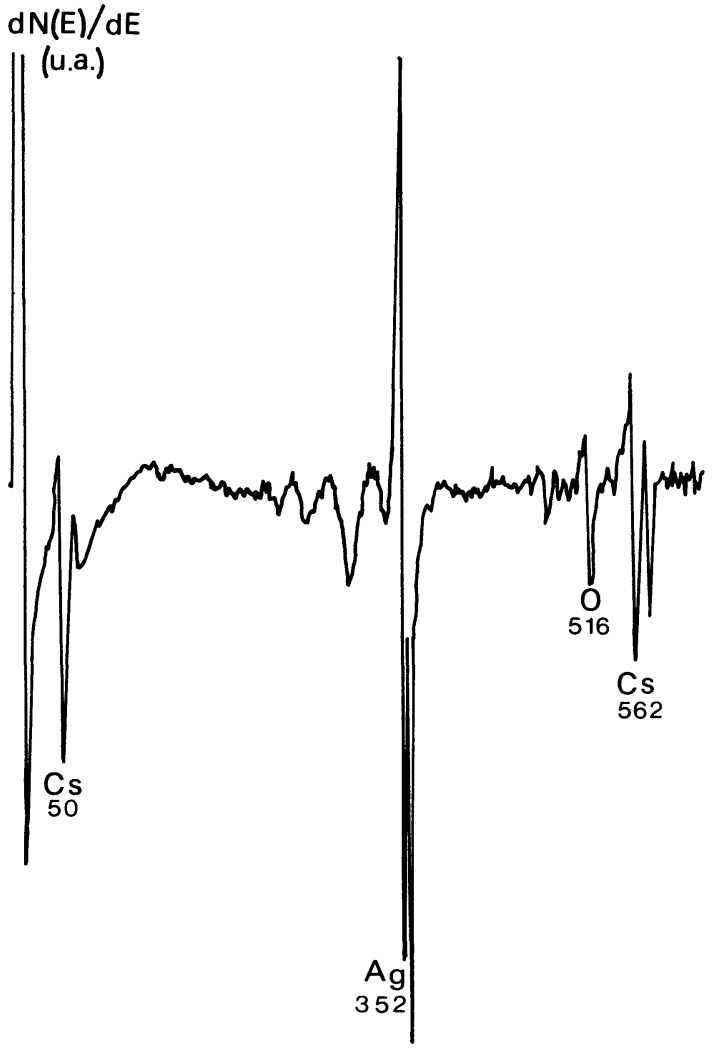

Fig. 8. - Spectre Auger d'une photocathode neuve, après 9 traitements $\mathrm{Cs}+\mathrm{O}_{2} .(V=2000 \mathrm{~V}-I=0,1 \mu \mathrm{A}-V$ modulation $=4 \mathrm{~V}$ - faisceau défocalisé.)

[Auger spectrum of a newly prepared Ag-Cs oxide photocathode, at the end of the 9th Cs $+\mathrm{O}_{2}$ cycle. (Operating conditions : $V=2000 \mathrm{~V}-I=0.1 \mu \mathrm{A}-V$ modulation $=4 \mathrm{~V}$ - defocused beam.)]

vation montre que l'épaisseur de la couche Cs-O est très faible, contrairement aux estimations de Yang et Bates [5] qui situent cette épaisseur entre 2 et $4 \mathrm{~nm}$, et confirme l'hypothèse selon laquelle les atomes d'oxygène et de césium seraient associés en une double couche avec atomes d'oxygène situés entre le substrat et les ions Cs [13-15].

L'amplitude du pic $\mathrm{Ag}(352 \mathrm{eV})$ restant pratiquement constante au cours des traitements successifs, et pour éliminer l'influence de fluctuations éventuelles du courant $I_{\mathrm{p}}$ au cours du temps nécessaire à l'élaboration de la couche d'oxyde de césium, nous avons étudié l'évolution des valeurs relatives des pics Cs $(50 \mathrm{eV})$, $\mathrm{Cs}(562 \mathrm{eV})$ et $\mathrm{O}(516 \mathrm{eV})$ rapportés au pic $\mathrm{Ag}(352 \mathrm{eV})$. En fonction du temps cumulé d'évaporation (pour le césium) ou d'oxydation (pour l'oxygène). Les variations de ces pics sont pratiquement linéaires (Figs. 9 et 10) - ce qui confirme de nouveau que l'épaisseur de la couche d'oxyde de césium est au plus égale à une monocouche en fin de traitement.

Les hauteurs relatives de pics Cs ou O, par rapport au pic $\mathrm{Ag}$, fluctuent légèrement lorsqu'on effectue des analyses en différents points de la surface, les écarts atteignant $10 \%$. Si l'on veut faire des comparaisons 


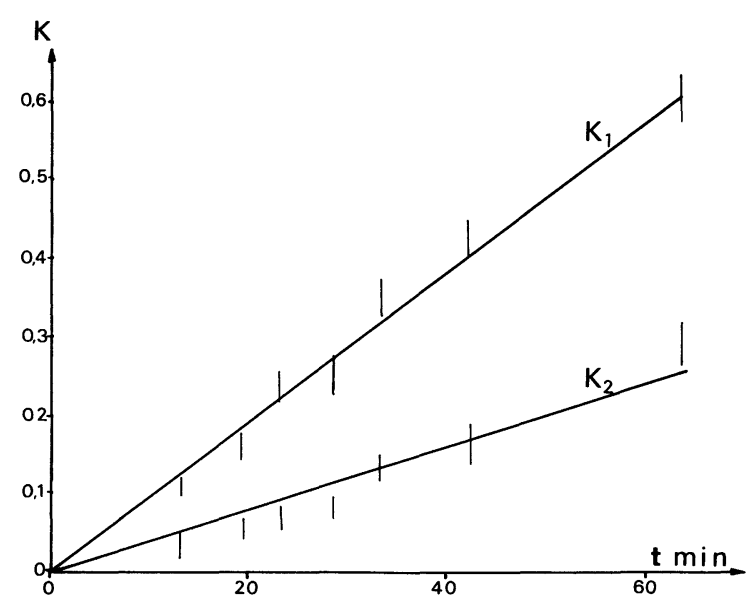

Fig. 9. - Evolution en fonction du temps d'exposition à l'oxygène des rapports $K_{1}$ (pic $\mathrm{Cs}(50 \mathrm{eV}) / \operatorname{pic~} \mathrm{Ag}(352 \mathrm{eV})$ ) et $K_{2}$ (pic Cs $(562 \mathrm{eV}) /$ pic Ag $\left.(352 \mathrm{eV})\right)$ au cours de la fabrication d'une photocathode.

[Variation as a function of the oxygen exposure of the Auger peaks ratios $K_{1}(\mathrm{Cs}(50 \mathrm{eV}) / \mathrm{Ag}(352 \mathrm{eV}))$ and $K_{2}(\mathrm{Cs}(562 \mathrm{eV}) / \mathrm{Ag}(352 \mathrm{eV}))$ during the activation process.]

quantitatives entre différents états de la couche, il faut donc tenir compte de ces écarts et raisonner sur des valeurs moyennes. C'est pourquoi nous avons effectué à chaque fois 7 analyses en 7 points différents répartis de façon aléatoire sur la surface.

Une photocathode optimisée et bien contrôlée au point de vue photoélectrique (seuil $\lambda_{\mathrm{s}}=1,2 \mu \mathrm{m}$ ) a été réalisée sans aucune analyse Auger.

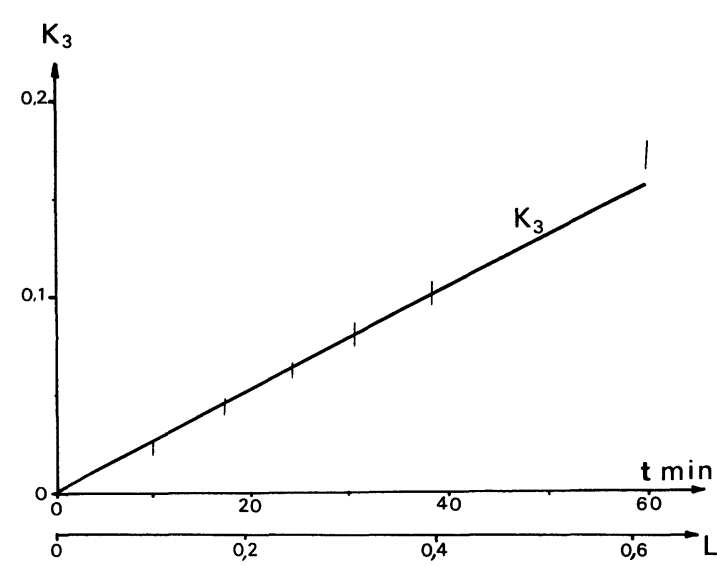

Fig. 10. - Evolution en fonction du temps d'exposition à l'oxygène, du rapport $K_{3}$ (pic $\mathrm{O}(516 \mathrm{eV}) /$ pic $\mathrm{Ag}(352 \mathrm{eV})$ ) au cours de la fabrication d'une photocathode.

[Variation, as a function of the oxygen exposure of the Auger peaks ratio $K_{3}(\mathrm{O}(516 \mathrm{eV}) / \mathrm{Ag}(352 \mathrm{eV}))$ during the activation process.]

Des analyses Auger ont ensuite été effectuées :

- après un vieillissement de $40 \mathrm{~h}$ (seuil $\lambda_{\mathrm{s}}=1,08 \mu \mathrm{m}$ ),

- après régénération par introduction d'oxygène,

- après un nouveau vieillissement de $60 \mathrm{~h}$,

- enfin après une nouvelle régénération.

Les résultats (hauteurs relatives de pics avec écarts types, seuils $\lambda_{s}$, et quantités d'oxygène ajoutées) sont portés dans le tableau I.

Par suite de la pollution entraînée par les analyses Auger, la régénération par introduction d'oxygène

Tableau I.

\begin{tabular}{|c|c|c|c|c|c|}
\hline & & & Hauteurs rela & tives des pics & \\
\hline & & Oxygène & Cés & um & \\
\hline Etat de la surfa & & $\mathrm{O}(517 \mathrm{eV})$ & Cs $(562 \mathrm{eV})$ & $\mathrm{Cs}(50 \mathrm{eV})$ & d'oxygène \\
\hline & $\lambda_{\mathrm{s}}(\mu \mathrm{m})$ & & & & \\
\hline Fin de fabrication & 1,20 & - & - & - & $\begin{array}{r}+0,580 \mathrm{~L} \\
1 \\
+3 \% \\
\end{array}$ \\
\hline $1^{\mathrm{e}}$ vieillissement $40 \mathrm{~h}$ & 1,08 & $\begin{array}{r}0,173 \pm 0,005 \\
+8 \%\end{array}$ & $\begin{array}{r}0,277 \pm 0,010 \\
+3 \%\end{array}$ & $\begin{array}{r}0,626 \pm 0,025 \\
+4 \%\end{array}$ & \\
\hline $1^{\mathrm{e}}$ régénération & 1,18 & $\begin{array}{r}0,187 \pm 0,008 \\
-17 \%\end{array}$ & $\begin{array}{r}0,285 \pm 0,006 \\
-14 \%\end{array}$ & $\begin{array}{r}0,652 \pm 0,020 \\
-3 \%\end{array}$ & $+0,020 \mathrm{~L}$ \\
\hline $2^{\mathrm{e}}$ vieillissement $60 \mathrm{~h}$ & 1,04 & $\begin{array}{r}0,154 \pm 0,014 \\
+5 \%\end{array}$ & $\begin{array}{r}0,244 \pm 0,026 \\
+10 \% \\
\end{array}$ & $\begin{array}{r}0,632 \pm 0,050 \\
-4 \%\end{array}$ & $\begin{array}{l}1 \\
+2 \%\end{array}$ \\
\hline $2^{\mathrm{e}}$ régénération & 1,15 & $0,162 \pm 0,010^{-}$ & $0,269 \pm 0,008$ & $0,602 \pm 0,028$ & $+0,014 \mathrm{~L}$ \\
\hline
\end{tabular}


n'est plus aussi parfaite que dans les expériences rapportées plus haut, effectuées sans analyse $\left(\lambda_{\mathrm{s}}\right.$ diminue d'une régénération à l'autre).

Il faut noter que les écarts observés entre les mesures effectuées à différents stades de vieillissement (ou de régénération) sont du même ordre de grandeur que ceux des mesures correspondant à différents points de la surface et se situent à la limite des possibilités de la méthode d'analyse.

\section{Discussion.}

Pour obtenir une photocathode ayant un seuil voisin de $\lambda_{\mathrm{s}}=1,2 \mu \mathrm{m}$, on peut réaliser à la surface d'un support métallique ou semiconducteur :

- soit une couche épaisse d'oxyde de césium riche en agrégats d'argent : c'est la cathode $S_{1}$, ou [Ag-O-Cs],

- soit une couche de quelques nanomètres d'oxyde de $\mathrm{Cs}$, formée essentiellement d'oxyde $\mathrm{Cs}_{2} \mathrm{O}$, avec éventuellement des inclusions de suboxyde $\mathrm{Cs}_{11} \mathrm{O}_{3}$ $[4,5,11]$,

- soit enfin, comme nous l'avons fait, une couche très mince contenant des atomes de césium et d'oxygène, optimisée à chaque cycle du traitement pour obtenir le travail de sortie minimum $W_{\mathrm{s}} \min$. Ce type de revêtement est utilisé pour l'obtention de cathodes à affinité négative sur substrat semiconducteur [6], en particulier la face $(100)$ du silicium $[15,17]$ et la face (110) de l'arséniure de gallium [18].

De nombreux travaux ont été consacrés à l'adsorption d'atomes alcalins à la surface de monocristaux métalliques ou semiconducteurs. Durant cette adsorption, le travail de sortie $W_{\mathrm{s}}$ commence par décroître, passe par un minimum $W_{\mathrm{s}}$ min puis croît et atteint une limite pratiquement égale au travail de sortie du métal alcalin massif, dès qu'une monocouche d'atomes alcalins est formée en surface. Les atomes adsorbés donnent - tout au moins sur des surfaces cristallines bien définies et sans défauts - des structures périodiques observables en diffraction d'électrons lents, ce qui permet de définir avec précision la densité superficielle d'atomes adsorbés. Les atomes de césium, qui ont un grand diamètre $(D \approx 0,5 \mathrm{~nm})$ ne peuvent pénétrer dans le réseau. Ils restent en surface sous forme partiellement ionisée, en donnant des dipôles avec les charges images, ce qui abaisse la valeur de $W_{s}$. $\mathrm{Au}$-delà de $W_{\mathrm{s}}$ min l'ionicité diminue par suite de l'interaction entre atomes voisins de Cs, qui retrouvent un caractère métallique lorsqu'une monocouche complète à structure hexagonale compacte est formée. L'adsorption de nouveaux atomes de Cs sur cette monocouche n'est plus possible à température ambiante, les forces de liaison étant trop faibles. Les atomes qui s'y fixent se réévaporent rapidement, ce qui explique que l'état final soit le même, quelle que soit la quantité de Cs évaporée sur le substrat. Le taux de recouvrement $\theta$, défini comme le rapport entre la densité superficielle d'atomes alcalins adsorbés et celle des atomes du substrat, est toujours inférieur à l'unité. Pour une monocouche saturée de Cs, on obtient par exemple $\theta_{\mathrm{Cs}_{\max }} \approx 0,50$ pour $\mathrm{W}(110)$ $[19,20], 0,43$ pour $\mathrm{W}(100)[12,21], 0,3$ pour $\mathrm{Ni}(100)$ [22] et 0,25 pour $\mathrm{Cu}(111)$ [23]. Dans tous les cas, le taux de recouvrement correspondant à $W_{\mathrm{s}}$ min est

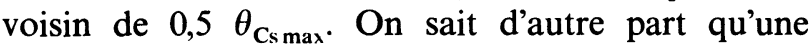
couche mince d'argent évaporée sur silice fondue est formée de monocristaux ayant en majorité leur surface orientée dans le plan (111) [24]. Les valeurs caractéristiques de $\theta_{\mathrm{Cs}}$ ne sont pas connues pour $\mathrm{Ag}$ mais par comparaison avec les valeurs relatives à $\mathrm{W}$, $\mathrm{Ni}$ et $\mathrm{Cu}$, on peut affirmer que $\theta_{\mathrm{Cs}_{\max }} \approx 0,3$ pour $\mathrm{Ag}(111)$ et 0,4 pour $\mathrm{Ag}(100)$. Le recouvrement pour lequel $W_{\mathrm{s}}=W_{\mathrm{s}}$ min serait alors de l'ordre de 0,5 monocouche d'atomes de $\mathrm{Cs}$, ce qui correspondrait à une épaisseur massique $d_{\mathrm{m}} \approx 0,14 \mathrm{~nm}$, en prenant $D=$ $0,47 \mathrm{~nm}$ pour le diamètre des atomes de Cs adsorbés [25].

Les électrons Auger de l'argent sont partiellement absorbés par cette couche de Cs. Nous avons observé que le pic $\mathrm{Ag}(352 \mathrm{eV})$ était atténué au maximum de $10 \%$. Si l'on admet [25] que le libre parcours inélastique d'électrons de $350 \mathrm{eV}$ dans Cs est $l \approx 1 \mathrm{~nm}$, l'épaisseur moyenne calculée $d$ serait voisine de $0,1 \mathrm{~nm}$, valeur inférieure à $d_{\mathrm{m}}$. On peut donc penser que le recouvrement en Cs n'est pas uniforme sur nos surfaces polycristallines d'argent, et qu'il existe des zones où les atomes de Cs ne peuvent se fixer.

L'adsorption d'oxygène sur des faces de monocristaux préalablement recouvertes d'atomes de Cs a été étudiée dans quelques cas particuliers W(112) [13], W(100) [12, 21], W(110) [20], Ni(100) [22], Cu(111) [23], $\mathrm{Si}(100)[7,15,17]$, en utilisant des techniques telles que la diffraction d'électrons lents, la mesure de $W_{s}$, et la désorption thermique, mais très peu de travaux font mention de spectres Auger, ce qui rend difficile une interprétation des variations de forme et de position de pics observés dans nos propres spectres.

Toutes les études montrent que le coefficient de collage de l'oxygène est pratiquement égal à l'unité sur une surface couverte d'atomes alcalins.

Pour un recouvrement donné $\theta_{\mathrm{Cs}}$, le travail de sortie décroît, passe par un minimum puis augmente lorsque le recouvrement en oxygène $\theta_{\mathrm{O}}$ croît. Le taux de recouvrement maximum en césium $\theta_{C_{s} \max }$ augmente avec $\theta_{0}$, par suite d'une décroissance du rayon des atomes de Cs adsorbés due à une ionisation croissante de ces atomes en présence des atomes d'oxygène ionisés négativement.

Le modèle le plus communément admis pour décrire la structure de la couche Cs-O est celui proposé par Chen [26] et Chen et Papageorgopoulos [27]. Les molécules $\mathrm{O}_{2}$ se dissocient au contact des atomes alcalins et les atomes $\mathrm{O}$ s'adsorbent en dessous des atomes de $\mathrm{Cs}$, ce qui renforce le moment des dipôles superficiels et abaisse $W_{\mathrm{s}}$. Les atomes d'oxygène de petit diamètre $(D \approx 0,12 \mathrm{~nm})$ peuvent pénétrer en partie dans la première couche d'atomes du substrat, 
et on peut avoir $\theta_{0} \max =1$. Les cycles alternés d'évaporation de $\mathrm{Cs}$ et d'introduction d'oxygène permettent d'obtenir le minimum absolu de $W_{\mathrm{s}}$ en adsorbant de nouveaux atomes de $\operatorname{Cs}\left(\theta_{\mathrm{Cs}}\right.$ augmente légèrement) et en complétant l'adsorption de $\mathrm{O}$. La double couche Cs-O est constituée d'atomes adsorbés, partiellement ionisés, et non d'oxyde de Cs déposé sur le substrat. Dans tous les cas, la densité d'atomes d'oxygène est supérieure à celle des atomes de Cs ce qui exclut la possibilité d'une couche homogène d'oxyde $\mathrm{Cs}_{2} \mathrm{O}$, Par exemple, dans le cas de Cs-O-W (100), on obtient $W_{\mathrm{s}}$ min pour $\theta_{\mathrm{Cs}}=0,25$ et $\theta_{\mathrm{o}}=1$ [14].

Un autre modèle a été proposé en 1983 pour les cathodes à affinité négative réalisées sur AsGa [18]. La réduction de $W_{\mathrm{s}}$ serait due à l'action d'un dipôle du type précédent (GaAs-O-Cs), recouvert d'une monocouche polarisée d'oxyde $\left(\mathrm{Cs}^{+} \mathrm{O}^{2-} \mathrm{Cs}^{+}\right)$. L'épaisseur totale de la couche active serait de l'ordre de $1 \mathrm{~nm}$.

A notre connaissance, l'adsorption d'oxygène sur des monocristaux d'Ag préalablement recouverts de Cs n'a pas encore été étudiée. Toutefois, une étude récente menée par XPS sur Ag polycristallin a montré que l'oxygène était adsorbé essentiellement sous forme atomique non entièrement ionisée [28].

L'adsorption d'oxygène sur faces propres de monocristaux d'Ag a fait l'objet de quelques travaux : $\operatorname{Ag}(110)$ [29-31], $\operatorname{Ag}(100)$ [32], $\operatorname{Ag}(111)[31,33]$.

L'examen de nos spectres Auger permet de souligner les points suivants :

- La couche superficielle a une épaisseur très faible, inférieure ou égale à une monocouche Cs-O. En effet, le pic $\mathrm{Ag}$ ( $352 \mathrm{eV}$ ) ne décroît à nouveau que de $10 \%$ entre le premier et le dernier cycle du traitement, et la croissance des pics Cs $(50 \mathrm{eV}), \mathrm{Cs}(562 \mathrm{eV})$ et $\mathrm{O}(516 \mathrm{eV})$ reste linéaire. La saturation de cette croissance, qui serait caractéristique de la formation d'une couche complète n'est pas atteinte en fin de traitement, en bon accord avec les résultats de Wang [21] relatifs à un monocristal W(100).

- Les atomes $\mathrm{Cs}$ et $\mathrm{O}$ sont liés à la surface sous forme d'adsorbats et non sous forme d'oxyde. Le pic Auger de l'oxygène reste fixe durant le traitement et dans toutes les expériences se situe vers $516 \pm 1 \mathrm{eV}$, et non vers $510 \mathrm{eV}$, valeur caractéristique des oxydes. D'autre part, la forme du pic est très différente de celle relative à des ions oxygène inclus dans un oxyde. La figure 11 montre les pics obtenus dans cette étude avec Cs-O adsorbés, et, dans une expérience annexe avec une couche d'aluminium recouverte d'oxyde $\mathrm{Al}_{2} \mathrm{O}_{3}$ pour laquelle le pic $\mathrm{O}$ se situe à $510 \mathrm{eV}$. Des résultats semblables ont été signalés pour la surface Cs-O-W(110), avec W oxydé ou non; l'écart en énergie était de $3,2 \mathrm{eV}$ entre les pics correspondant aux deux états de $\mathrm{O}$ [25].

Une étude menée à l'aide de la spectroscopie de photoélectrons (XPS) sur l'adsorption de O sur $\mathrm{K}-\mathrm{Fe}(110)$ a également montré qu'il n'y avait pas
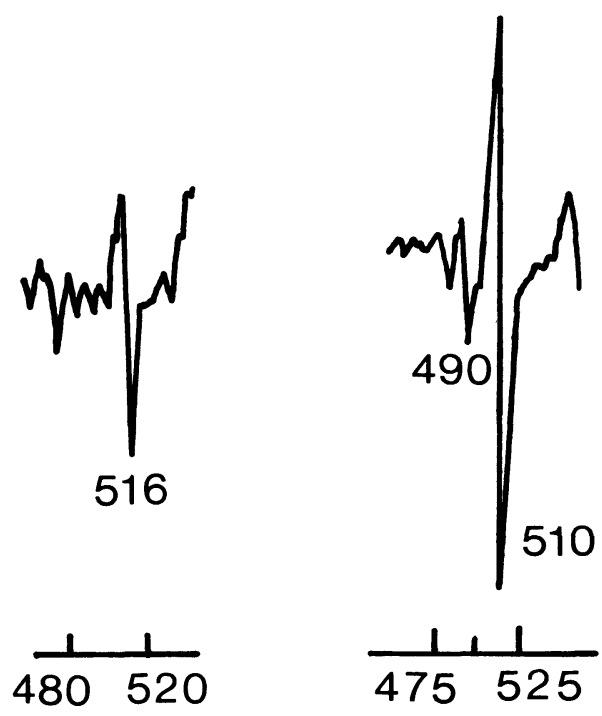

$-\mathrm{a}$ -

$-b-$

Fig. 11. - Forme des pics Auger de l'oxygène - (a) : oxygène adsorbé sur les photocathodes $(E \simeq 516 \mathrm{eV})-(\mathrm{b})$ : oxygène de $\mathrm{Al}_{2} \mathrm{O}_{3}(E \simeq 510 \mathrm{eV})$.

[Shape of oxygen Auger signals - (a) : oxygen in the photocathode $(E \simeq 516 \mathrm{eV})-(\mathrm{b})$ : oxygen in $\mathrm{Al}_{2} \mathrm{O}_{3}$ $(E \simeq 510 \mathrm{eV})$.]

formation d'oxyde $\mathrm{K}_{2} \mathrm{O}$ tant que $\theta_{\mathrm{O}}$ restait inférieur à l'unité et $\theta_{\mathrm{K}}$ inférieur à $\theta_{\mathbf{K}_{\text {max }}}=0,31$, les atomes de $\mathrm{K}$ étant adsorbés entre la couche de $K$ et la première couche $\mathrm{Fe}$ [34]. De même, l'adsorption d'oxygène sur Cs-Si(100) avec $\theta_{0} \leqslant 1$ n'entraîne pas la formation d'oxyde $\mathrm{SiO}$ ou $\mathrm{SiO}_{2}$ [15].

On peut toutefois remarquer, durant l'adsorption d'oxygène, l'apparition sur le pic Auger Ag (352 eV) d'un nouveau pic de faible amplitude d'énergie $E=335 \mathrm{eV}$. Ce pic pourrait correspondre à la formation en certains points de la surface de la couche d'argent, qui d'après [24] comporte de nombreux défauts, de quelques molécules d'oxyde d'argent.

Nos couches actives Cs-O formées sur argent sont donc différentes de celles obtenues avec GaAs et décrites dans [18] : elles sont plus minces, et dépourvues d'oxyde $\mathrm{Cs}_{2} \mathrm{O}$, ce qui nous incite à adopter pour nos photocathodes le modèle du dipôle simple Ag-O-Cs.

- L'adsorption d'oxygène change profondément l'ionicité des atomes de Cs. Les deux pics Auger du Cs, initialement situés à 562 et $574 \mathrm{eV}$ se déplacent durant le traitement d'environ $3 \mathrm{eV}$ vers de plus faibles énergies, en se déformant légèrement.

Le vieillissement des photocathodes est dû à la désorption d'une faible partie des atomes d'oxygène. L'examen du tableau I (si on exclut la dernière valeur relative à $\mathrm{Cs}(562 \mathrm{eV})$ ), semble indiquer que le vieillissement des photocathodes qui s'accompagne d'un accroissement du travail de sortie et de la sensibilité $S_{\lambda}$ à $0,65 \mu \mathrm{m}$, est provoqué par une désorption 
simultanée d'oxygène et de césium, et que la régénération correspond au processus inverse, puisqu'il y a variation des hauteurs des pics Cs et $O$. Il faut donc imaginer que l'adsorption d'atomes d'oxygène pendant la régénération est capable d'initier une adsorption d'atomes de Cs. Ce phénomène semble peu vraisemblable, car le césium évaporé en surplus pendant la formation de la photocathode plusieurs heures auparavant doit être transformé en oxyde sur les parois de l'enceinte (effet « Getter » bien connu des alcalins).

Une explication peut être trouvée dans un article de Levine [15] sur l'obtention de l'affinité négative à la surface de monocristaux (100) de silicium. Un accroissement de la hauteur des pics $\mathrm{Cs}(47 \mathrm{eV})$ et Cs $(560 \mathrm{eV})$ pendant l'adsorption d'oxygène a été observé. Il est dû, non à un accroissement de concentration en Cs, mais à l'augmentation de la rétrodiffusion des électrons Auger du Cs par la sous-couche d'atomes d'oxygène dont la densité superficielle s'accroît. Cette hypothèse a été vérifiée en bombardant la surface avec des électrons d'énergie primaire $47 \mathrm{eV}$ et en mesurant le coefficient de rétrodiffusion en présence, et en l'absence d'oxygène adsorbé [15].

On peut donc affirmer que le vieillissement est dû seulement à une désorption partielle des atomes d'oxygène, ce qui explique que la régénération puisse être obtenue simplement par une brève exposition à l'oxygène. Le même phénomène a été observé dans le cas de l'affinité négative de la face (100) du silicium [15].

Le départ des atomes d'oxygène s'accompagne d'une remontée de $W_{\mathrm{s}}$ min. Il s'agit donc d'atomes adsorbés sur les faces cristallines ayant à la fin du traitement Cs-O le plus bas travail de sortie $W_{\mathrm{s}} \min$. Les nombreuses études menées sur l'abaissement de $W_{\mathrm{s}}$ par adsorption d'alcalins et d'oxygène montrent que $W_{\mathrm{s}}$ min est d'autant plus faible que le travail de sortie du substrat est plus élevé au départ. Pour l'argent, c'est la face (111) qui a le $W_{\mathrm{s}}$ le plus élevé [34, 35], car c'est la face la plus dense.

Dans nos couches minces polycristallines, le seuil photoélectrique va donc dépendre de l'adsorption de l'oxygène sur les faces (111) qui sont nombreuses, mais très perturbées [24]. Or, on sait que si l'adsorption d'oxygène est facile et très stable sur les faces peu denses (100) et (110) [20,30,32], il n'en est pas de même pour la face (111) où l'adsorption est faible et peu reproductible [30]. Les atomes d'oxygène ne peuvent pénétrer que très faiblement au centre des mailles du réseau d'argent par suite de la faible distance entre atomes et d'après certains auteurs [33], l'adsorption ne pourrait avoir lieu qu'en présence de défauts à l'échelle atomique. Le départ des atomes d'oxygène les moins liés aux atomes d'Ag, c'est-à-dire les atomes adsorbés sur les faces (111), va faire croître $W_{\mathrm{s}} \mathrm{min}$, la valeur finale de $W_{\mathrm{s}}$ étant alors fixée par les dipôles stables existant sur les faces (100) et (110).

Nos couches polycristallines d'Ag permettent donc d'obtenir un seuil photoélectrique très intéressant grâce à la présence des faces (111), mais ce seuil n'est pas stable. D'autre part, la sensibilité au voisinage du seuil $\lambda_{\mathrm{s}}$, pour une couche fraîchement préparée, dépend de l'importance relative des faces (111), et du taux d'adsorption de l'oxygène sur ces faces.

Cette sensibilité va varier avec les propriétés cristallines des couches d'argent, et donc la technique de préparation, ce qui peut expliquer l'écart de sensibilité observé entre nos couches, et celles de G. Hincelin préparées dans un autre bâti [2].

On peut donc envisager de réaliser à l'avenir des cathodes sur monocristaux (111) d'argent, mais on n'aura plus la possibilité d'y exciter des plasmons de surface pour accroître le rendement photoélectrique. Avec des films minces dans lesquels cette excitation est possible, il faudra alors chercher à accroître au maximum la proportion de microcristaux orientés (111). Dans les deux cas, un moyen de fixer les atomes d'oxygène sur le réseau d'argent devra être trouvé. On peut penser :

- modifier la formation de la couche Cs-O en faisant adsorber $\mathrm{Cs}$ sur une surface d'Ag légèrement oxydée,

- accroître le nombre de défauts par un bombardement ionique de la surface [35],

- effectuer des recuits des couches Cs-O-Ag vers $550^{\circ} \mathrm{C}$, de tels recuits ayant permis de stabiliser des cathodes à affinité négative $[18,37]$.

Des études approfondies sont encore nécessaires pour parvenir à ce résultat.

\section{Bibliographie}

[1] Hincelin, G. et Septier, A., C. R. Heb. Séan. Acad. Sci. 284B (1977) 173.

[2] Hincelin, G. et SePtier, A., Proc. 7th Int. Vacuum Congr. and 3rd Int. Conf. on Sol. Surf., Vienna (1977), p. 1269

[3] Gautreau, L., Mémoire Ingénieur CNAM, Paris (1982).

[4] Ebbinghaus, G., Brown, W., Simon, A., Berresheim, K., Phys. Rev. Lett. 37 (1976) 1770.

[5] Yang, S. J., Bates, C. W., Appl. Phys. Lett. 36 (1980) 675 .
[6] Bell, R. L., Negative Electron Affinity Devices (Clarendon Press, Oxford) 1973.

[7] Gundry, P. M., Holtom, R., Leverett, V., Surf. Sci. 43 (1974) 647.

[8] Kan, H., Nakamura, T., Katsuno, H., Hagino, M., Sukegawa, T., Appl. Phys. Lett. 34 (1979) 545.

[9] Kretschmann, E., Z. Phys. 241 (1971) 319.

[10] Gerlach, R. L., Rhodin, T. N., Surf. Sci. 19 (1970) 403.

[11] Uebbing, J. J., James, L. W., J. Appl. Phys. 41 (1970) 4505 . 
[12] Papageorgopoulos, G. A., Chen, J. M., Surf. Sci. 39 (1973) 283.

[13] Papageorgopoulos, G. A., Chen, J. M., J. Vac. Sc. Techn. 9 (1971) 570.

[14] Papageorgopoulos, G. A., Chen, J. M., Surf. Sci. 39 (1973) 313.

[15] Levine, J. D., Surf. Sci. 34 (1973) 90.

[16] Sommer, A. H., Photoemission Materials (Wiley, NewYork) 1968, Chap. 10.

[17] Goldstein, B., Surf. Sci. 35 (1973) 227.

[18] Su, C. Y., Spicer, W. E., Lindau, I., J. Appl. Phys. 54 (1983) 1413.

[19] Fedorus, A. G., Naumovets, A. G., Surf. Sci. 21 (1970) 426.

[20] Desplat, J. L., Papageorgopoulos, G. A., Surf. Sci. 92 (1980) 97.

[21] Chen-Show Wang, J. Appl. Phys. 48 (1977) 1477.

[22] Papageorgopoulos, G. A., Chen, J. M., Surf. Sci. 52 (1975) 40

[23] Lindgren, S. A., Wallden, L., Phys. Rev. B 22 (1980) 5967.

[24] Dujardin, M. M., Theye, M. L., J. Phys. Chem. Sol. 32 (1971) 2033.
[25] Papageorgopoulos, G. A., Desplat, J. L., Surf. Sci. 92 (1980) 119.

[26] Chen, J. M., J. Appl. Phys. 41 (1970) 5008.

[27] Chen, J. M., Papageorgopoulos, G. A., Surf. Sci. 26 (1971) 499.

[28] АучоOв, M., Hedge, M. S., Surf. Sci. 133 (1983) 516.

[29] BackX, C. De Groot, C.P.M., Biloen, P., Surf. Sci. 104 (1981) 300.

[30] Engelhardt, H., Menzel, D., Surf. Sci. 57 (1976) 591.

[31] Martin, R. L., Hay, P. J., Surf. Sci. 130 (1983) L-283.

[32] Kitson, M., Lambert, R. M., Surf. Sci. 109 (1981) 60.

[33] Albers, H., Van der Wal, W. J. J., Bootsma, G. A., Surf. Sci. 68 (1977) 47.

[34] Pirug, G., Brodén, G., Bonzel, H. P., Surf. Sci. 94 (1980) 323.

[35] Chelvayoham, M., Mee, C. H. B., J. Phys. C 15 (1982) 2305.

[36] Albers, H., Droog, J. M. M., Bootsma, G. A., Surf. Sci. 64 (1977) 1.

[37] Stocker, B. J., Surf. Sci. 47 (1975) 501. 